\title{
Developmental control of CFTR: from bioinformatics to novel therapeutic approaches
}

\author{
Catherine M. Greene ${ }^{1}$ and Dominik Hartl ${ }^{2}$
}

Affiliations: ${ }^{1}$ Dept Medicine, Royal College of Surgeons in Ireland, Dublin, Ireland. ${ }^{2}$ CF Research Group, Children's Hospital, Dept I, University of Tübingen, Tübingen, Germany.

Correspondence: Catherine M. Greene, Dept Medicine, RCSI Education and Research Centre, Beaumont Hospital, Beaumont, Dublin 9, Ireland. E-mail: cmgreenedrcsi.ie

0 @ERSpublications

miRNA-binding blocker oligonucleotides offer an appealing option for developing tools to correct CFTR function http://ow.ly/AjS8a

Celebrating the 25th anniversary of identifying the genetic cause of cystic fibrosis (CF), tremendous progress has been made in the understanding and the treatment of this complex, but still fatal disease [1]. Despite advances, several key questions about the pathomechanisms of CF remain poorly understood. For instance, the precise genetic and epigenetic mechanisms that regulate expression and stability of the cystic fibrosis transmembrane conductance regulator (CFTR) gene remain incompletely defined.

CFTR gene expression is a carefully controlled process that is spatially and temporally regulated. Notwithstanding the extensive research that has been carried out on CFTR over the past few decades, developmental control of CFTR expression is still poorly understood [2]. Transcription of CFTR starts at distinct sites depending on the tissue or developmental stage, and is positively regulated by a selection of transcription factors, including CCAAT-enhancer-binding protein (C/EBP) proteins and Forkhead Box A (FOXA) factors. In addition, CFTR is controlled post-transcriptionally by microRNAs (miRNAs). miRNAs are regulatory factors involved in most biological processes and it is becoming increasingly clear that they play a key role in the development and manifestations of CF lung disease [3-13]. These small non-coding RNAs act post-transcriptionally to inhibit protein production. Their involvement in the pathogenesis of CF lung disease stems from the fact that their expression is altered in vivo in the CF lung due to intrinsic and extrinsic factors; to date, defective chloride ion conductance, endoplasmic reticulum stress, inflammation and infection have been implicated in altering endogenous miRNA expression in this setting.

With their recent study, VIART et al. [14] now substantially expand our understanding of how CFTR expression is regulated (fig. 1). The authors identified regulatory elements that participate in CFTR downregulation, particularly FOXA1, FOXA2 and C/EBP $\alpha$. Using bioinformatics, they further identified four new AU-rich elements (ARE) and experimentally tested their roles in regulation of CFTR expression. Following a series of intricate studies, ARE-5698 was found to play a role in destabilising CFTR mRNA. Regarding miRNAs predicted to bind to the CFTR 3'UTR, miR-101 had the strongest repressive effect. By comparing miRNA expression profiles of adult and fetal lungs, three specific miRNAs (miR-145, miR-150 and miR-451) were found to have a temporal effect, being significantly upregulated in the adult lung and, therefore, contributing to downregulation of CFTR.

With a view to therapeutics that might enhance CFTR expression, miRNA-binding blocker oligonucleotides (MBBOs; also known as target site blockers/protectors or miRNA masks) were designed. The function of an $\mathrm{MBBO}$ is to prevent binding of a miRNA to one specific mRNA target. Here MBBOs for several miRNAs

Received: July 302014 | Accepted: July 312014

Conflict of interest: None declared.

Copyright OERS 2015 
a)

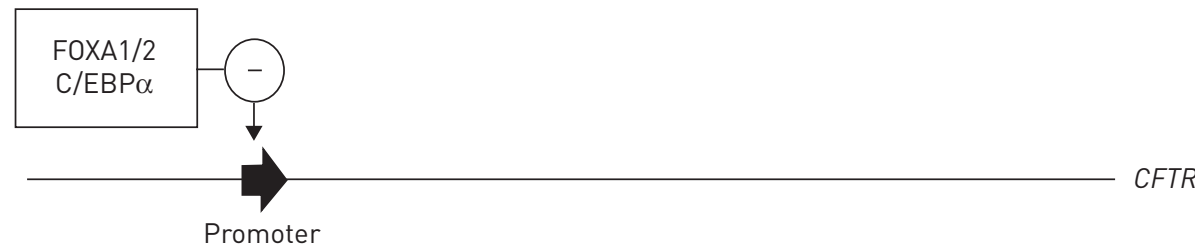

b)

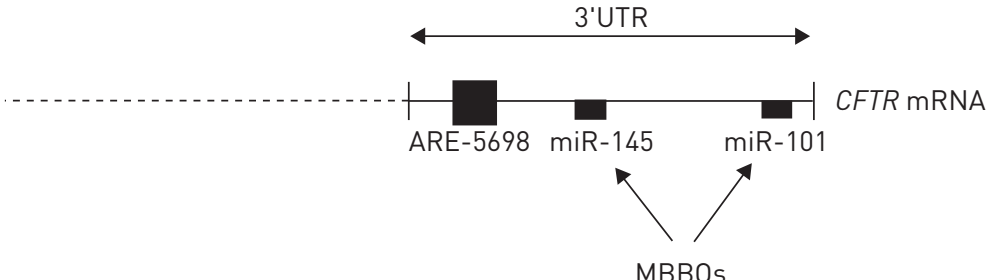

FIGURE 1 Transcriptional and post-transcriptional regulation of cystic fibrosis transmembrane conductance regulator (CFTR). In the adult lung, a) the transcription factors FOXA1/2 and $\mathrm{C} / \mathrm{EBP} \alpha$ negatively regulate CFTR gene expression, while b) ARE-5698 destabilises CFTR mRNA and miR-145 and miR-101 block CFTR expression. MBBOs targeting the miR-145 and miR-101 sites can restore CFTR expression. FOXA: Forkhead Box A; C/EBP: CCAAT-enhancer-binding protein; ARE: AU-rich elements; MBBOs: microRNA-binding blocker oligonucleotides.

that bind the CFTR $3^{\prime} \mathrm{UTR}$ were tested in vitro and ex vivo using non-CF and p.Phe508del homozygous CF primary human nasal epithelial cells grown at an air-liquid interface. MBBOs targeting the miR-145 and miR-101 sites were most effective, as they stabilised CFTR mRNA and enhanced CFTR protein expression. Finally, both MBBOs significantly enhanced CFTR function in CFBE41o- cells.

This report adds significantly to our existing knowledge regarding miRNA regulation of CFTR [5-10, 13] and the developing field of miR-based CFTR therapeutics [15]. The data confirms previous studies implicating miR-145 and miR-101 as important modulators of CFTR expression $[5,6,8,9]$ and build on them by demonstrating how MBBOs based on these miRNAs can affect CFTR gene expression and CFTR protein function. The authors suggest that the MBBOs may be developed as tools for CFTR correction in people with CF. This is an appealing option given that MBBOs are less likely to have off-target effects compared with some other miRNA inhibition strategies [16].

Although there are a series of novel findings from this paper, the work also raises a number of important questions for further investigation. The CF lung is inherently associated with microbial colonisation. Whether infection impacts upon the newly described transcriptional and post-transcriptional mechanisms controlling CFTR expression remains elusive. This will be particularly important for the further development of MBBOs as therapeutics for CF. Previous studies have reported how infection and inflammation affect the expression of miRNAs, including those that regulate CFTR $[9,13]$. This suggests that higher than normal levels of MBBOs may be required to ensure an inhibitory effect on endogenous miRNA activity. Nonetheless, it will be a very exciting development when miRNA-modulating drugs for CF advance to the stage of clinical studies. A second point worth considering is the relevance of the findings of VIART et al. [14] beyond CF. Separate sets of studies have clearly shown that cigarette smoke affects CFTR and miRNA expression. Although the mechanisms responsible for altered CFTR expression in, for example, the chronic obstructive pulmonary disease lung are not restricted to miRNA-mediated effects [17], miR-101 in particular is known to be increased by cigarette smoke extracts and directly impacts on CFTR expression [8]. Finally, it is becoming increasingly evident that dysfunctional CFTR as a result of cigarette smoking can contribute to the pathophysiology of a range of extrapulmonary disease conditions, such as chronic pancreatitis, male infertility and cachexia [18]. Expanding upon the findings from airway epithelial cells reported here into non-lung cells that express CFTR may yield novel therapies for these disease conditions beyond CF.

\section{References}

Mall MA, Hartl D. CFTR: cystic fibrosis and beyond. Eur Respir J 2014; 44: 1042-1054.

McCarthy VA, Harris A. The CFTR gene and regulation of its expression. Pediatr Pulmonol 2005; 40: 1-8.

Oglesby IK, Bray IM, Chotirmall SH, et al. miR-126 is downregulated in cystic fibrosis airway epithelial cells and regulates TOM1 expression. J Immunol 2010; 184: 1702-1709.

4 Bhattacharyya S, Balakathiresan NS, Dalgard C, et al. Elevated miR-155 promotes inflammation in cystic fibrosis by driving hyperexpression of interleukin-8. J Biol Chem 2011; 286: 11604-11615.

5 Gillen AE, Gosalia N, Leir SH, et al. MicroRNA regulation of expression of the cystic fibrosis transmembrane conductance regulator gene. Biochem J 2011; 438: 25-32. 
Megiorni F, Cialfi S, Dominici C, et al. Synergistic post-transcriptional regulation of the cystic fibrosis transmembrane conductance regulator (CFTR) by miR-101 and miR-494 specific binding. PLoS One 2011; 6: e26601.

7 Ramachandran S, Karp PH, Jiang P, et al. A microRNA network regulates expression and biosynthesis of wild-type and $\Delta$ F508 mutant cystic fibrosis transmembrane conductance regulator. Proc Natl Acad Sci USA 2012; 109: $13362-13367$.

8 Hassan F, Nuovo GJ, Crawford M, et al. MiR-101 and miR-144 regulate the expression of the CFTR chloride channel in the lung. PLoS One 2012; 7: e50837.

9 Oglesby IK, Chotirmall SH, McElvaney NG, et al. Regulation of cystic fibrosis transmembrane conductance regulator by microRNA-145, -223 , and -494 is altered in $\Delta$ F508 cystic fibrosis airway epithelium. J Immunol 2013; 190: 3354-3362.

10 Amato F, Seia M, Giordano S, et al. Gene mutation in microRNA target sites of CFTR gene: a novel pathogenetic mechanism in cystic fibrosis? PLoS One 2013; 8: e60448.

11 Megiorni F, Cialfi S, Cimino G, et al. Elevated levels of miR-145 correlate with SMAD3 down-regulation in cystic fibrosis patients. J Cyst Fibros 2013; 12: 797-802.

12 Rupani H, Sanchez-Elsner T, Howarth P. MicroRNAs and respiratory diseases. Eur Respir J 2013; 41: 695-705.

13 Ramachandran S, Karp PH, Osterhaus SR, et al. Post-transcriptional regulation of cystic fibrosis transmembrane conductance regulator expression and function by microRNAs. Am J Respir Cell Mol Biol 2013; 49: 544-551.

14 Viart V, Bergougnoux A, Bonini J, et al. Transcription factors and miRNAs that regulate fetal to adult CFTR expression change are new targets for cystic fibrosis. Eur Respir J 2015; 45: 116-128.

15 Amato F, Tomaiuolo R, Nici F, et al. Exploitation of a very small peptide nucleic acid as a new inhibitor of miR-509-3p involved in the regulation of cystic fibrosis disease-gene expression. Biomed Res Int 2014; 2014: 610718.

16 Hassan T, McKiernan PJ, McElvaney NG, et al. Therapeutic modulation of miRNA for the treatment of proinflammatory lung diseases. Expert Rev Anti Infect Ther 2012; 10: 359-368.

17 Rasmussen JE, Sheridan JT, Polk W, et al. Cigarette smoke-induced $\mathrm{Ca}^{+}$release leads to cystic fibrosis transmembrane conductance regulator (CFTR) dysfunction. J Biol Chem 2014; 289: 7671-7681.

18 Raju SV, Jackson PL, Courville CA, et al. Cigarette smoke induces systemic defects in cystic fibrosis transmembrane conductance regulator function. Am J Respir Crit Care Med 2013; 188: 1321-1330. 